\title{
Antisense oligonucleotide targeting CD39 improves anti-tumor T cell immunity
}

\footnotetext{
* Correspondence: frank.jaschinski@secarna.com; alfred.zippelius@usb.ch

${ }^{\dagger}$ Abhishek S. Kashyap, Tamara Thelemann, Frank Jaschinski and Alfred

Zippelius contributed equally to this work.

2Secarna Pharmaceuticals GmbH, Planegg/Martinsried, Germany

${ }^{1}$ Cancer Immunology, Department of Biomedicine, University Hospital Basel,

Basel, Switzerland

Full list of author information is available at the end of the article
}

(c) The Author(s). 2019 Open Access This article is distributed under the terms of the Creative Commons Attribution 4.0 International License (http://creativecommons.org/licenses/by/4.0/), which permits unrestricted use, distribution, and reproduction in any medium, provided you give appropriate credit to the original author(s) and the source, provide a link to the Creative Commons license, and indicate if changes were made. The Creative Commons Public Domain Dedication waiver (http://creativecommons.org/publicdomain/zero/1.0/) applies to the data made available in this article, unless otherwise stated. 


\section{Background}

Local immunosuppression in the tumor microenvironment is a hallmark of many cancers. Reinvigoration of $\mathrm{T}$ cell function by checkpoint blockade can result in striking clinical responses, but is effective only in a minority of patients. Immunosuppressive pathways operational in tumors significantly impact the efficacy of immunotherapy. The adenosine pathway is one of these immunosuppressive pathways. CD39 and CD73 are ectonucleotidases which act in concert to degrade ATP to immunosuppressive adenosine [1-4]. Binding of adenosine to A2A or A2B receptors on T cells and natural killer (NK) cells results in dampened proliferation and cytolytic functions leading to immunosuppression. Adenosine induces alternate polarization of tumor associated macrophages (TAMs) towards immunosuppressive M2-like TAMs capable of secreting increased amounts of IL-10 and reduced pro-inflammatory cytokines $[4,5]$. Mice lacking CD39 show improved tumor rejection [1], making this pathway a promising therapeutic target [6]. Dying cancer cells release ATP which may enhance anti-tumor immune responses e.g. through recruitment and activation of dendritic cells (DCs), macrophages and their precursors [7, 8]. Binding of ATP to $\mathrm{P}_{2} \mathrm{X}_{7}$ receptors on DCs results in DC activation and the release of pro-inflammatory cytokines such as IL-1 $\beta$ or IL-18 [9]. These cytokines in turn activate NK cells, T cells, and macrophages and enhance their proliferation and cytolytic functions [5]. Co-expression of CD73 by tumor cells or tumor-associated stromal cells leads to further degradation to adenosine that accumulates in the tumor microenvironment [4]. Taken together, targeting CD39 will decrease the degradation of ATP and therefore result in increased levels of immune stimulatory extracellular ATP. This will concurrently lead to the suppression of adenosine through the prevention of AMP generation, the substrate of CD73.

CD39 is widely expressed by different immune cells such as monocytes, neutrophils, macrophages, B lymphocytes, DCs, as well as some subsets of NK cells, and T cells [3]. Tregs express CD39 and CD73, which enable them to generate adenosine leading to immunosuppression. In addition, enhanced CD39 expression has been described in various cancer cells of kidney, lung, testicular and thyroid tumors as well as in lymphoma [7].

In the current study we targeted CD39 expression with locked nucleic acid (LNA) Gapmer antisense oligonucleotides (ASOs). LNA Gapmers are ASOs with a length of typically 14-17 nucleotides. They contain a central "gap" of DNA monomers flanked by LNA-modified nucleotides. LNA modifications result in increased target affinity. The central DNA "gap" recruits RNase $\mathrm{H}$, which cleaves the target RNA upon binding. LNA Gapmers have fully phosphorothioated (PTO) backbones, which ensure resistance to enzymatic degradation [10]. In contrast to earlier chemical modifications, LNA Gapmers do not require transfection reagents or conjugations for efficient gene silencing in vitro $[11,12]$, a process called gymnosis. In vivo unformulated and unconjugated LNA Gapmers achieve specific target knockdown in several tissues including tumors after systemic administration [12].

Here we demonstrate that unformulated human (h)CD39 specific ASOs achieved potent target knockdown in vitro, reduced degradation of extracellular ATP by T cells, and reverted growth suppression in T cells caused by treatment with ATP. In syngeneic mouse tumor models, systemic treatment with murine (m)CD39-specific ASO resulted in potent knockdown of CD39 expression in specific immune cell populations, namely Tregs and TAMs, as well as in a reduction of the frequency of intratumoral Tregs. Moreover, tumor growth was significantly reduced when CD39-specific ASO was combined with an anti-PD-1 antibody.

\section{Methods}

\section{Antisense oligonucleotides}

15-, 16- and 17-mer ASOs were selected based on the human NM_001776 or murine NM_001304721 CD39 mRNA (encoded by the ENTPD1 gene). Main criterion for sequence selection was selectivity to avoid undesired off-target effects. LNA modified Gapmers were ordered from Exiqon or Eurogentec and dissolved in $\mathrm{H}_{2} \mathrm{O}$ (stock concentration: $1 \mathrm{mM}$ ). Antisense oligonucleotides were added to cells without the use of a transfection reagent in vitro and without any delivery system in vivo.

Sequences of ASOs and control oligonucleotides used in the study:

\begin{tabular}{|c|c|c|c|}
\hline ASO ID & Length & Sequence & Description \\
\hline$\overline{\mathrm{A} 04019 \mathrm{H}}$ & 15 & $\begin{array}{l}+G^{*}+T^{*}+A^{*} A^{*} G^{*} C^{*} C^{*} C^{*} T^{*} G \\
{ }^{*} A^{*} T^{*}+G^{*}+T^{*}+T\end{array}$ & Human specific ASO \\
\hline $\mathrm{A04040 \textrm {H }}$ & 16 & $\begin{array}{l}+G^{*}+T^{*}+T^{*} T^{*} G^{*} T^{*} G^{*} T^{*} G^{*} A \\
{ }^{*} G^{*} A^{*} G^{*} C^{*}+T^{*}+T\end{array}$ & $\begin{array}{l}\text { Human specific ASO, } \\
\text { used for in vitro experiments }\end{array}$ \\
\hline $\mathrm{A} 04042 \mathrm{H}$ & 17 & $\begin{array}{l}+T^{*}+G^{*}+C^{*} C^{*} A^{*} G^{*} A^{*} G^{*} T^{*} G \\
{ }^{*} C^{*} C^{*} T^{*} G^{*}+A^{*}+T^{*}+C\end{array}$ & Human specific ASO \\
\hline $\mathrm{A} 04044 \mathrm{H}$ & 17 & $\begin{array}{l}+T^{*}+T^{*}+A^{*} C^{*} G^{*} T^{*} T^{*} C^{*} A^{*} C \\
{ }^{*} T^{*} A^{*} C^{*} C^{*}+T^{*}+T^{*}+C\end{array}$ & Human specific ASO \\
\hline $\mathrm{A} 04045 \mathrm{H}$ & 17 & $\begin{array}{l}+C^{*}+A^{*}+C^{*} T^{*} T^{*} A^{*} C^{*} G^{*} T^{*} T \\
{ }^{*} C^{*} A^{*} C^{*} T^{*}+A^{*}+C^{*}+C\end{array}$ & Human specific ASO \\
\hline A04011MR & 16 & $\begin{array}{l}+A^{*}+G^{*}+T^{*} A^{*} A^{*} T^{*} C^{*} C^{*} A \\
{ }^{*} C^{*} C^{*} C^{*} A^{*}+T^{*}+A^{*}+G\end{array}$ & $\begin{array}{l}\text { Mouse specific } \\
\text { ASO, used for } \\
\text { in vivo experiments, } \\
\text { named: "CD39 ASO" } \\
\text { in the text }\end{array}$ \\
\hline Control oligo 1 & 18 & $\begin{array}{l}+C^{*}+G^{*}+T^{*} T^{*} T^{*} A^{*} G^{*} G^{*} C \\
{ }^{*} T^{*} A^{*} T^{*} G^{*} T^{*} A^{*}+C^{*}+T^{*}+T\end{array}$ & $\begin{array}{l}\text { Control oligonucleotide, } \\
\text { reference: PMID: } 26072406 \text {, } \\
\text { used for in vitro and } \\
\text { in vivo experiments }\end{array}$ \\
\hline Control oligo 2 & 17 & $\begin{array}{l}+T^{*}+C^{*}+T^{*} A^{*} T^{*} C^{*} G^{*} T^{*} G \\
{ }^{*} A^{*} T^{*} G^{*} T^{*} T^{*}+T^{*}+C^{*}+T\end{array}$ & $\begin{array}{l}\text { Control oligonucleotide, } \\
\text { used for in vitro experiments }\end{array}$ \\
\hline
\end{tabular}

+ indicates LNA-modified nucleotides and ${ }^{*}$ indicates PTO linkages 


\section{Cell culture medium and supplements}

RPMI supplemented with antimycotic-antibiotic (100x) and sodium pyruvate $(100 \mathrm{x})$ were used at $1 \%$ and heat inactivated $\left(30 \mathrm{~min}\right.$ at $\left.56^{\circ} \mathrm{C}\right) \mathrm{FBS}$ was used at $10 \%$ for cell culture experiments (RPMIfs). Cell culture reagents were obtained from GIBCO.

\section{Blood samples}

PBMC were derived from Buffy Coats purchased from Blutspendedienst des Bayerischen Roten Kreuzes gemeinnützige GmbH; Hauptverwaltung München; Vertrieb; Herzog-Heinrich-Straße 2; 80336 München or obtained from leukapheresis products.

\section{Mice}

C57BL/6 and Balb/c mice were bred in-house at University Hospital Basel, Switzerland. In case of unavailability, mice were also obtained from Janvier Labs (France). Animals were housed under specific pathogen-free conditions. All animal experiments were performed in accordance with Swiss federal regulations. Sex-matched littermates at 8-12 weeks of age at start of experiments were used.

\section{Quantigene mRNA expression analysis}

Target expression on mRNA level was determined using bDNA assay (QuantiGene SinglePlex Assay Kit 96-Well plate format and QuantiGene Sample Processing Kit for cultured cells, Thermo Fisher Scientific). The following probe sets were used: human ENTPD1 (SA-11803); human HPRT1 (SA-10030); mouse ENTPD1, (SB-13732); mouse HPRT1 (SB-15463). All reagents were purchased from Affymetrix/Thermo Fisher Scientific.

\section{FACS staining for surface proteins for human samples}

Cells were spun down at $500 \mathrm{~g}$ for $5 \mathrm{~min}$, and washed in FACS buffer (1x PBS, 5\% FBS) followed by incubation for $25 \mathrm{~min}$ at $4{ }^{\circ} \mathrm{C}$ in $50 \mu \mathrm{l}$ FACS buffer per well in 96-well U-bottom plates containing the respective antibodies (anti- human CD8 (clone RPA-T8), anti-human CD4 (clone RPA-T4), anti-human CD39 (clone A1), mouse IgG,K isotype control and 7-AAD (all from BioLegend). Subsequently, cells were washed twice with FACS buffer and analyzed on a NovoCyte Flow Cytometer (ACEA Biosciences, Inc.).

hCD39 protein expression in human $\mathrm{CD} 8^{+}$or $\mathrm{CD} 4^{+} \mathrm{T}$ cells upon oligonucleotide treatment

$\mathrm{CD}^{+}{ }^{+}$and $\mathrm{CD}^{+} \mathrm{T}$ cells were separately isolated from PBMCs using MACS (Miltenyi, according to the manufacturer's instructions). $\mathrm{CD}^{+}$or $\mathrm{CD}^{+} \mathrm{T}$ cells $(100,000$ per well) were plated on anti-CD3-coated $(2 \mu \mathrm{g} / \mathrm{ml}$; clone OKT3; eBioscience) 96-well U-bottom plates in RPMIfs supplemented with anti-CD28 $(2 \mu \mathrm{g} / \mathrm{ml}$; clone CD28.2; eBioscience) and IL-2 (60 IU/ml; Peprotec) and treated with $5 \mu \mathrm{M}$ of oligonucleotides for a total treatment time of six days without the use of a transfection reagent. Activation medium and oligonucleotides were replaced after three days. As mock control, cells were cultivated in activation medium without oligonucleotide. On day six after start of treatment, cells were transferred to uncoated 96-well U-bottom plates and cultivated in cell culture medium supplemented with IL-2 $(20 \mathrm{IU} / \mathrm{ml})$ in the absence of oligonucleotides. Cells were split 1:2 every third day. hCD39 protein expression was analyzed on day three, six and eleven after removal of oligonucleotides by flow cytometry.

\section{Labelling of cells with proliferation dye}

$\mathrm{T}$ cells were isolated as described above. Cells were washed twice with PBS, resuspended and adjusted to $2 \mathrm{x}$ of the desired final concentration in PBS (pre-warmed to room temperature). A $20 \mu \mathrm{M}$ solution of cell proliferation dye eFluor ${ }^{\mathrm{Tn}} 450$ (eBioscience) in PBS was prepared (pre-warmed to room temperature) and mixed 1:1 with the $2 \mathrm{x}$ cell suspension while gently vortexing. Cells were incubated for $10 \mathrm{~min}$ at $37^{\circ} \mathrm{C}$ in the dark. The labelling reaction was stopped by adding 4-5 volumes of cold complete medium (containing 10\% FBS) and cells were incubated on ice for $5 \mathrm{~min}$. Cells were washed 3 times with complete medium before further culturing.

Quantification of extracellular ATP levels in cell supernatants of oligonucleotide-treated human $\mathrm{CD}^{+} \mathrm{T}$ cells

$\mathrm{CD} 8^{+} \mathrm{T}$ cells were isolated from PBMC as described above. 100,000 cells per well were plated on anti-CD3-coated 96 well U-bottom plates in activation medium (as described above) and treated with $5 \mu \mathrm{M}$ of oligonucleotides for a total treatment time of six days without the use of a transfection reagent. Activation medium and oligonucleotides were replaced after three days. As mock control, cells were cultivated in activation medium without oligonucleotide. Six days after treatment start, cell culture medium was supplemented with ATP (SIGMA-Aldrich) at a concentration of $2 \mu \mathrm{M}$. After an incubation time of $30 \mathrm{~min}$, residual ATP concentration in cell culture supernatants or cell-free medium was determined using the ATP Bioluminescence Assay Kit HSII (Roche) according to the manufacturer's protocol.

\section{Assessment of extracellular ATP on proliferation of oligonucleotide-treated human $\mathrm{CD}^{+} \mathrm{T}$ cells}

$\mathrm{CD}^{+} \mathrm{T}$ cells were isolated and labelled with cell proliferation dye as described above. Cells (100,000 per well) were plated on anti-CD3-coated 96 well U-bottom plates in activation medium and treated with oligonucleotides at a concentration of $5 \mu \mathrm{M}$ for a total treatment time of five days without the use of a transfection reagent. Activation medium and oligonucleotides were replaced after 
three days. On day three and day four after start of oligonucleotide treatment, $400 \mu \mathrm{M}$ of ATP or vehicle were added to cells. The following day, hCD39 protein expression, proliferation, and absolute numbers of $\mathrm{CD} 8^{+}$ $\mathrm{T}$ cells were analyzed by flow cytometry (123count eBeads from eBioscience were used to obtain absolute counts). Proliferation index was calculated using the formula: $\sum_{0}^{i} N_{i} / \sum_{0}^{i} N_{i} / 2$, whereas $\mathrm{i}$ is the generation number and $\mathrm{N}$ is the absolute cell count in the respective generation.

\section{In vivo tumor challenge and treatment protocol}

C57BL/6 mice were injected subcutaneously into the right flank with 500,000 syngeneic murine MC38 colorectal adenocarcinoma cells (kindly provided by Thomas Wirth, Medizinischen Hochschule Hannover) suspended in phenol red-free DMEM (without additives). Once tumors reached an average volume of $60-80 \mathrm{~mm}^{3}$ (day 12-15), mice were injected intraperitoneally with $200 \mu \mathrm{l}$ of PBS suspended solutions of CD39 ASO at indicated doses, non-targeting control oligonucleotide (control oligo 1) $(100 \mathrm{mg} / \mathrm{kg})$ or left untreated. On day 9 post first compound injection, mice were euthanized and tumors, and in selected cases tumor draining lymph nodes were excised and processed for FACS analyses as outlined below. For tumor growth experiments EMT6 (obtained from ATCC) murine breast cancer cells were injected into the mammary gland of female Balb/c mice. Once tumors reached an average volume of $80 \mathrm{~mm}^{3}$ (Day 8), mice were injected intraperitoneally with $200 \mu \mathrm{l}$ of PBS suspended solutions of CD39 ASO $(20 \mathrm{mg} / \mathrm{kg})$, non-targeting control oligonucleotide (control oligo 1) $(20 \mathrm{mg} / \mathrm{kg})$ and $/$ or mouse anti-PD-1 (12.5 mg/kg) (RPM114, rat IgG2a, BioXCell) at indicated timepoints. Tumor volume was calculated according to the formula: $\mathrm{D} / 2 * \mathrm{~d} * \mathrm{~d}$, with $\mathrm{D}$ and $\mathrm{d}$ being the longest and shortest tumor diameter in $\mathrm{mm}$, respectively.

\section{Phenotypic characterization of tumor infiltrating and lymph node cells by flow cytometry}

Tumors were harvested from tumor bearing mice and minced using razor blades followed by digestion with accutase (PAA), collagenase IV (Worthington), hyaluronidase (Sigma), and DNAse type IV (Sigma) for 60 min at $37^{\circ} \mathrm{C}$ with constant shaking. The cell suspensions were filtered using a cell strainer $(70 \mu \mathrm{m})$. Lymph node cells were isolated by mashing using the end of a $1 \mathrm{~mL}$ syringe. Cells were filtered through a $70 \mu \mathrm{m}$ nylon mesh. Red blood cells (RBCs) were lysed using RBC lysis buffer (eBioscience). Single cell suspensions derived from tumor and lymph nodes were blocked with rat anti-mouse FcyIII/II receptor (CD16/ CD32) blocking antibodies ("Fc-Block") and stained with live/dead cell-exclusion dye (Zombie UV dye; Biolegend). The cells were then incubated with fluorophore-conjugated antibodies directed against cell surface antigens, washed and resuspended in FACS buffer (PBS $+2 \%$ FBS). For intracellular antigens (FoxP3), cells stained with cell surface antibodies were fixed (IC fix, eBioesceince) and permeabilized (Perm buffer; eBioscience) prior to incubation with antibodies directed against intracellular antigens. Cell populations were analyzed on a BD Fortessa. Cells were discriminated using the following combination of cell markers after gating on single cells (discriminated by FSC-A and FSC-H) and excluding non-viable cells (Live/Dead negative). TAMs were de-

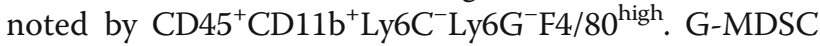
were $\mathrm{CD} 45^{+} \mathrm{CD} 11 \mathrm{~b}^{+} \mathrm{Ly} 6 \mathrm{G}^{+}$and $\mathrm{M}-\mathrm{MDSC}$ were $\mathrm{CD} 45^{+}$

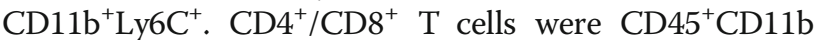
${ }^{-} \mathrm{CD}^{+} \mathrm{CD} 4^{+}$or $\mathrm{CD} 8^{+}$. Tregs were $\mathrm{FOXP3}^{+} \mathrm{CD} 25^{+} \mathrm{CD} 4^{+} \mathrm{T}$ cells. B cells were $\mathrm{CD} 45^{+} \mathrm{CD} 11 \mathrm{~b}^{-} \mathrm{CD} 3^{-} \mathrm{CD} 19^{+}$. Tumor cells were noted as $\mathrm{CD} 45^{-}$.

\section{Statistical analysis}

Statistical analysis was performed by GraphPad Prism 7.0 (GraphPad Software). If applicable, results are represented as mean $+/-$ SD. Pairwise comparisons were analyzed using two-tailed student's t-test and grouped analyses were performed using one-way non-parametric ANOVA with multiple comparison with Tukey's post hoc test. $p$-values $\leq 0.05\left(^{(*)} ; p \leq 0.01{ }^{(* *)}\right) ; p \leq 0.001{ }^{(* * *)}$; $\left.p \leq 0.0001{ }^{(* * * * *)}\right)$ were considered significant.

\section{Results}

\section{CD39 protein expression is reduced in human $\mathrm{CD}^{+}$and} $\mathrm{CD}^{+} \mathrm{T}$ cells after hCD39-specific ASO treatment

ASOs with specificity for hCD39 were initially screened for target mRNA suppression in HDLM-2 cells without the use of a transfection reagent, a human Hodgkin lymphoma cell line with high endogenous expression of CD39. The most potent candidates were further tested in dose-response experiments. Figure 1a depicts the concentration dependency of target knockdown for the ASOs with the highest activity. The corresponding IC50 values are shown in Additional file 1: Table S1. The most potent hCD39-specific ASO (A04040H, IC50 $25.28 \mathrm{nM}$ ) was selected for subsequent experiments.

The potency of $\mathrm{A} 04040 \mathrm{H}$ was investigated in human $\mathrm{T}$ cells. To this end, human $\mathrm{T}$ cells were exposed to A $04040 \mathrm{H}$ for six days without the use of a transfection reagent and, upon washing, subsequently cultured in the absence of $\mathrm{A} 04040 \mathrm{H}$. CD39 protein expression was determined three, six and eleven days after oligonucleotide removal. CD39 protein expression in $\mathrm{T}$ cells remained downregulated up to 6 days after 

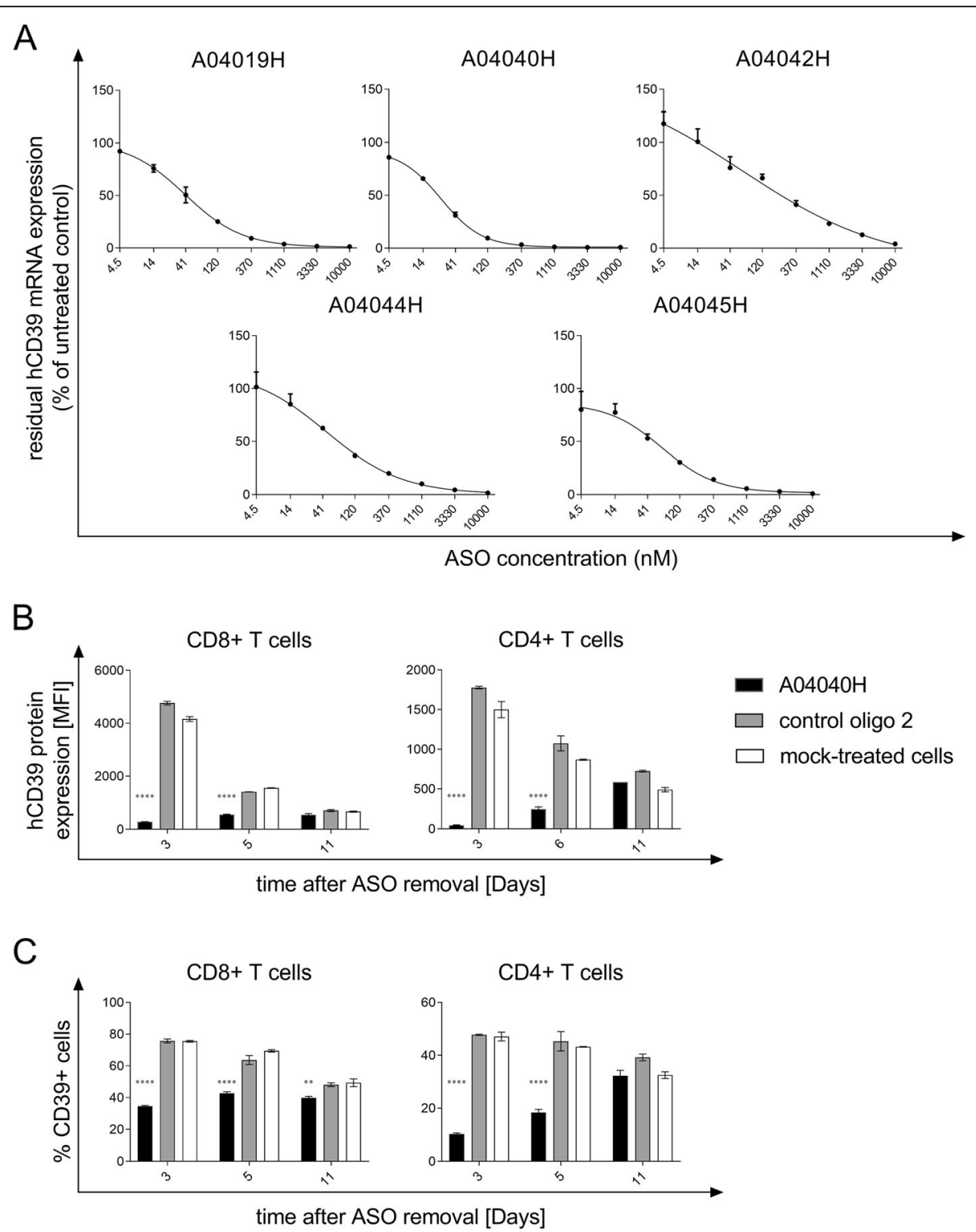

Fig. 1 Selection of hCD39-specific ASO and assessment of target knockdown efficiency. (a) HDLM-2 cells were treated for three days with the indicated concentrations of the respective antisense oligonucleotide without the use of a transfection reagent. hCD39 mRNA expression values were normalized to expression of the housekeeping gene HPRT1. Residual hCD39 mRNA expression relative to untreated cells (set to 100\%) is depicted. Depicted is the mean of triplicate wells +/- SD. (b) and (c) Human anti-CD3, anti-CD28, and IL-2 activated CD8 ${ }^{+}$and CD4 $4^{+}$T cells were treated with $5 \mu \mathrm{M}$ of the hCD39-specific ASO A04040H or the control oligo 2 for a total treatment time of six days without the use of a transfection reagent. Thereafter, oligonucleotides were removed and cells were re-plated on plates not coated with anti-CD3. hCD39 protein expression was analyzed by flow cytometry three, six and eleven days after oligonucleotide removal. hCD39 protein expression is depicted as mean fluorescence intensity (MFI) and was calculated by subtracting the MFI of hCD39 by the MFI of unspecific isotype control (b) or as \% CD39 cells of alive cells (c). Data is shown as mean of duplicates +/-SD. Asterisks indicate significant differences compared to control oligo treatment within each time point

removal of $\mathrm{A} 04040 \mathrm{H}$ as measured by median fluorescence intensity (Fig. 1b) and \%CD39 ${ }^{+}$cells (Fig. 1c), respectively. Treatment with the control oligo 2 that has no sequence complementarity to any human or murine mRNA had no inhibitory effect on hCD39 protein expression as compared to mock-treated cells (Fig. $1 \mathrm{~b}$ and $\mathrm{c}$ ).

\section{hCD39-specific ASO prevents extracellular ATP} degradation by human $\mathrm{CD}^{+}{ }^{+}$cells

We assessed the effects of hCD39-specific ASO on the capacity of $\mathrm{CD}^{+} \mathrm{T}$ cells and tumor cells (here Burkitt's lymphoma cells) to degrade extracellular ATP, the substrate of CD39. Cells were treated with $\mathrm{A} 04040 \mathrm{H}$ for six days without the use of a transfection reagent. ATP was 
added to cell culture supernatants or cell-free medium for $30 \mathrm{~min}$ after which ATP levels (and CD39 protein expression) were determined. As observed above, treatment with $\mathrm{A} 04040 \mathrm{H}$ led to strong suppression of CD39 protein expression compared to control oligo 1 or mock-treated cells (Fig. 2a and Additional file 2: Figure S1A). In cells treated with $\mathrm{A} 04040 \mathrm{H}$, the decreased expression of CD39 correlated with significantly increased levels of extracellular ATP compared to control oligo 1 or mock treatment (Fig. $2 \mathrm{~b}$ and Additional file 2: Figure S1B). This suggests that the hCD39-specific ASO prevents CD39-mediated degradation of extracellular ATP by targeting CD39 expression.

\section{hCD39-specific ASO reverts the impairment of T cell} proliferation and viability caused by ATP by-products

We next investigated the effects of $\mathrm{A} 04040 \mathrm{H}$ on $\mathrm{CD}^{+}$ $\mathrm{T}$ cell proliferation in the presence or absence of extracellular ATP. A04040H treatment of $\mathrm{CD}^{+} \mathrm{T}$ cells potently suppressed CD39 protein expression (Fig. 3a). In the absence of extracellular ATP no significant differences in proliferation index (Fig. $3 \mathrm{~b}$ ) or absolute cell numbers (Fig. 3c) were observed compared to control oligo 2 or mock treatment. As expected, ATP supplementation reduced the proliferation (Fig. $3 \mathrm{~b}$ ) and reduced absolute numbers (Fig. 3c) of control oligo 2 or mock-treated $\mathrm{CD}^{+} \mathrm{T}$ cells. This was entirely rescued by treatment with $\mathrm{A} 04040 \mathrm{H}$. We furthermore observed no impact on cell viability upon exposure of A04040H-treated T cells with ATP (Fig. 3d). In contrast, there was a reduction in cell viability (non-significant) when ATP was added to control oligo 2-treated cells and a significant reduction in cell viability when ATP was added to mock-treated cells. In summary, these results reveal that $\mathrm{A} 04040 \mathrm{H}$ treatment rescues CD39-induced suppression of $\mathrm{T}$ cell proliferation and cell viability, most likely by inhibiting ATP degradation.

mCD39-specific ASO preferentially downregulates CD39 expression in murine tumor infiltrating Tregs and TAMs We next utilized syngeneic tumor models to assess the in vivo effects of CD39 ASOs. Upon subcutaneous injection of MC38 colon adenocarcinoma cells, CD39 protein expression was initially assessed on tumor cells and tumor infiltrating immune populations. Myeloid cells, namely TAMs, as well as T lymphocytes expressed higher levels of CD39 compared to B cells and tumor cells (Fig. 4a). CD4 $4^{+}$Tregs as well as PD-1 expressing $\mathrm{CD}^{+}$and $\mathrm{CD}_{4}^{+} \mathrm{T}$ cell subsets had higher CD39 expression compared to non-Tregs and PD-1 negative cells, respectively (Fig. 4b). Of note, CD39 expression was higher in intratumoral $\mathrm{T}$ cells compared to $\mathrm{T}$ cells derived from the tumor draining lymph nodes (TDLN) (Fig. 4b).

To assess target downregulation and efficacy in vivo, murine-specific CD39 ASOs (Additional file 3: Table S2) were tested in vitro in mouse cancer cell line A20 (B cell lymphoma) from which the most potent mCD39-specific ASO A04011MR was selected as candidate (Additional file 4: Figure S2) for in vivo experiments. Systemic administration (intraperitoneal, i.p.) of
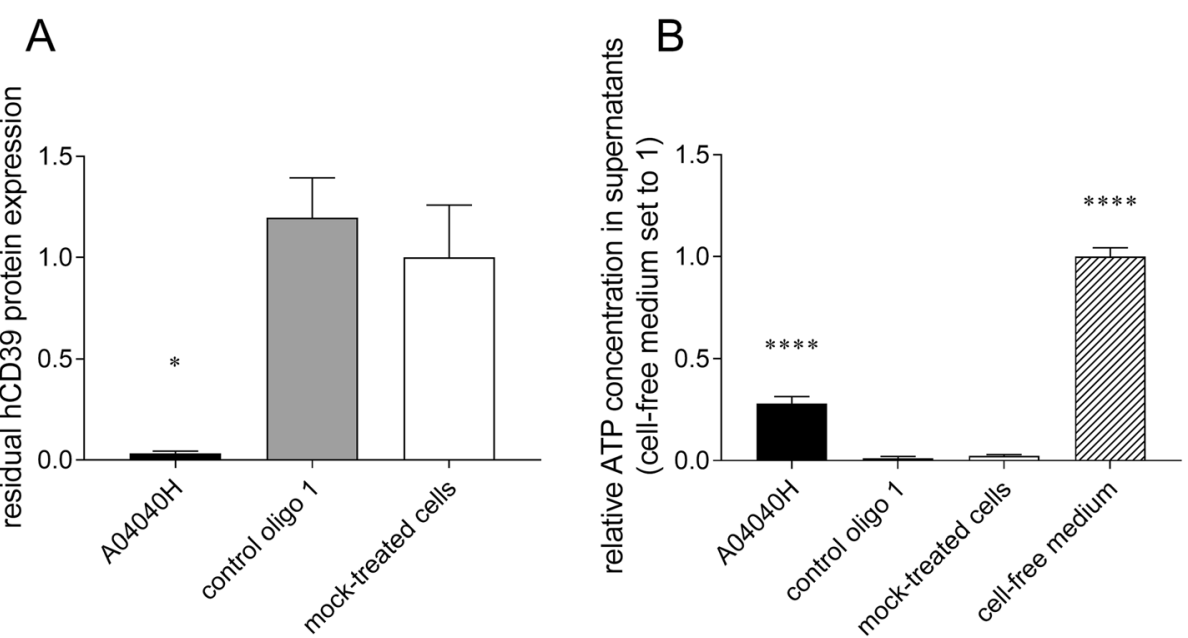

Fig. 2 Decrease in CD39 expression and extracellular ATP accumulation in human CD8 ${ }^{+} \mathrm{T}$ cells in presence of hCD39-specific ASO. Human antiCD3, anti-CD28, and IL-2 activated CD8 ${ }^{+}$T cells were treated with $5 \mu \mathrm{M}$ of the hCD39 specific ASO A04040H or the control oligo 1 for a total treatment time of six days. hCD39 protein expression was analyzed by flow cytometry. (a) Residual hCD39 expression of oligonucleotide-treated cells relative to mock-treated cells (set to 1). (b) After six days of ASO treatment, $2 \mu \mathrm{M}$ of ATP were added to cells or cell-free medium. ATP concentration in cell supernatants was determined after $30 \mathrm{~min}$ of incubation with ATP and is presented relative to cell-free medium (set to 1). Data is shown as mean of triplicates +/- SD. Asterisks indicate significant differences compared to control oligo treatment 
A

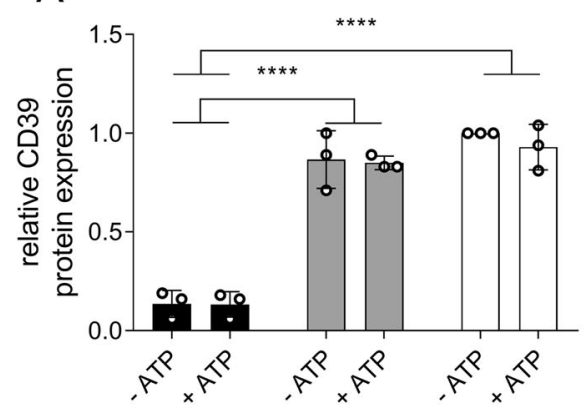

C

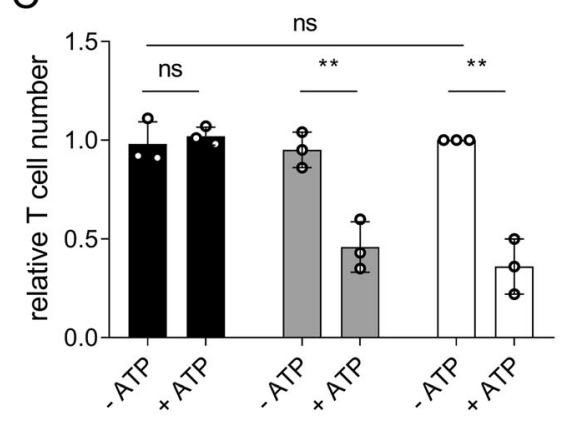

B

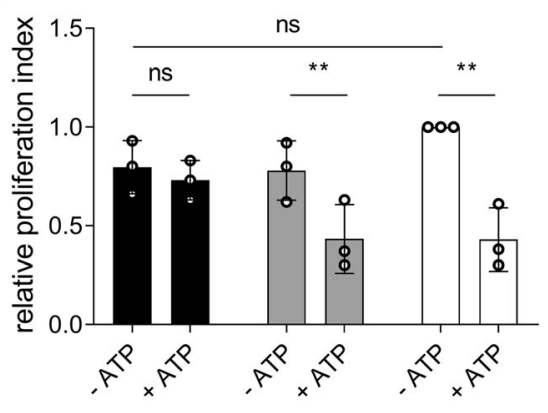

D

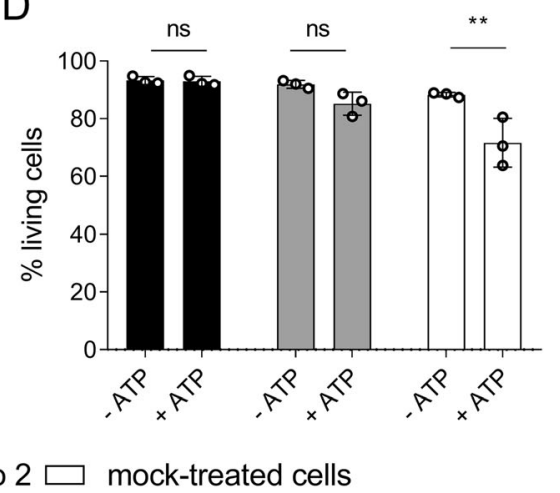

Fig. 3 Improved human CD8 $8^{+} T$ cell proliferation in the presence of hCD39-specific ASO and extracellular ATP. Human CD8 $8^{+} \mathrm{T}$ cells were labelled

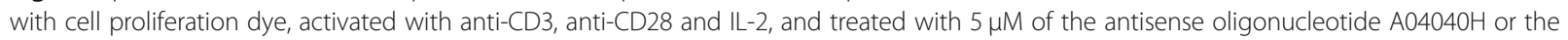
control oligo 2 for a total treatment time of five days. Subsequently, $400 \mu \mathrm{M}$ of ATP was added to cells on day three and day four after start of oligonucleotide-treatment. On day five after start of oligonucleotide treatment, (a) hCD39 protein expression, (b) proliferation index, (c) relative cell numbers of $\mathrm{CD}^{+} \mathrm{T}$ cells relative to mock-treated cells without ATP (set as 1) as wells as (d) \% living cells were analyzed using flow cytometry. Cells were derived from two different donors, processed on three different dates. Bar graphs depict the mean of three independent experiments run in triplicates +/- SD. Asterisks indicate significant differences between the respective conditions

the mCD39-specific ASO in MC38 tumor bearing mice resulted in a specific and dose-dependent downregulation of CD39 protein in tumor infiltrating $\mathrm{CD} 4^{+}$Tregs as well as TAMs (Fig. 4c, d and Additional file 5: Figure S3A). Greater than $50 \%$ of target downregulation was observed in Tregs, and TAMs, even at the low dose of $20 \mathrm{mg} / \mathrm{kg}$ of the ASO. CD39 expression in non-Tregs, $\mathrm{CD}^{+} \mathrm{T}$ cells, either PD-1 $1^{+}$or PD-1 $1^{-}$, as well as tumor cells remained unchanged (Fig. 4c and e and Additional file 5: Figure S3B). When assessed for percentage of CD39 positive $\mathrm{T}$ cell populations, we observed a significant decrease in the number of $\mathrm{CD} 39$ expressing $\mathrm{CD}^{+} \mathrm{T}$ cells as well as $\mathrm{CD}^{+}$and Tregs in tumors of mice treated with CD39 ASO compared to control oligo 1 (Additional file 5: Figure S3C, D, F and G). Similar to that observed for CD39 surface expression (assessed by MFI), number of $\mathrm{CD} 39^{+} \mathrm{CD}^{+} \mathrm{T}$ cells in CD39 ASO mice remained non-significantly different to control oligo 1 (Additional file 5: Figure S3E). Together, these data suggest that systemically administered mCD39-specific ASO enters distant tumors and induces cell type specific downregulation of the target and the target cell populations.

\section{mCD39-specific ASO targets intratumoral Tregs and improves $\mathrm{CD}^{+}:$Treg ratio}

Tumor infiltrating $\mathrm{T}$ cell populations were analyzed by flow cytometry after treatment with mCD39-specific or control oligo 1. A dose-dependent reduction in Treg cell numbers (and frequency) was observed in tumors of mice treated with mCD39-specific ASO while non-Treg $\mathrm{CD}^{+} \mathrm{T}$ cell numbers remained unchanged (Fig. 5a, b). Particularly, we noticed that the decrease in Treg frequency was positively correlated with the extent of CD39 expression (Fig. 5c). CD8 ${ }^{+} \mathrm{T}$ cell frequency did not change upon treatment with the ASO (Additional file 6: Figure S4A). As a consequence of altered Treg cell frequency, the $\mathrm{CD} 8^{+} \mathrm{T}$ cell to Treg ratio was substantially higher in the tumors of mCD39 ASO treated mice (Fig. 5d). This indicates a potential skewing from an immunosuppressive to a pro-inflammatory tumor microenvironment. Accordingly, $\mathrm{CD}^{+} \mathrm{T}$ cells in the mCD39-specific ASO treated tumors expressed higher levels of PD-1 (Fig. 5e) and CD25 (Additional file 6: Figure S4B), which may likely reflect increased $\mathrm{T}$ cell activation. Compared to the control oligo 1 group, the majority of $\mathrm{CD}^{+} \mathrm{T}$ cells in 

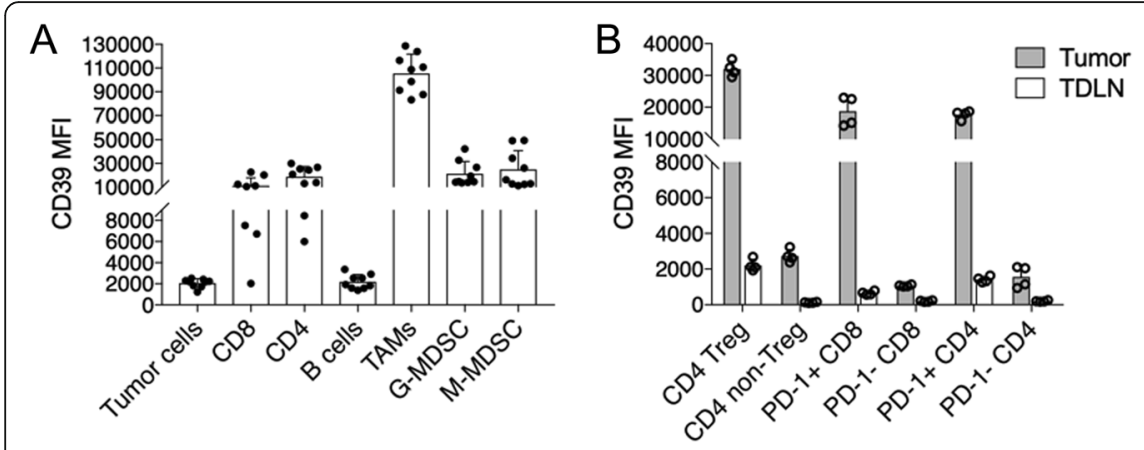

C CD4

$\mathrm{D}$

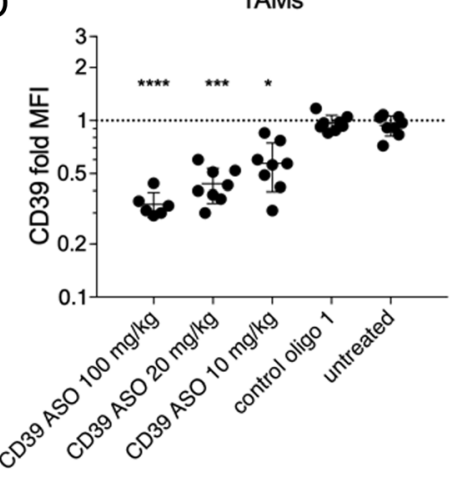

$\mathrm{E}$

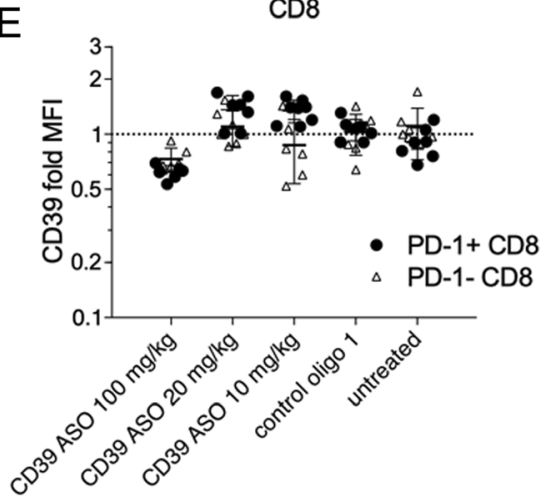

Fig. 4 CD39 expression on various murine tumor infiltrating lymphocyte and myeloid populations. (a) CD39 expression on live CD45+ immune cells or CD45- tumor cells from freshly digested MC38 murine tumors (around 100 to $200 \mathrm{~mm}^{3}$ ) of untreated mice was assessed by flow

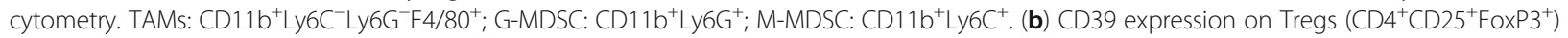
and non-Tregs $\left(C D 4^{+} \mathrm{CD}_{25}{ }^{-}\right.$FoxP3 $\left.3^{-}\right)$as well as PD-1 ${ }^{+}$or PD- $1^{-}$CD4 and CD8 T cells was assessed in tumors and tumor draining-lymph nodes (TDLN) of untreated mice. (c)-(e) Mice bearing palpable tumors $\left(50-80 \mathrm{~mm}^{3}\right.$ ) were injected i.p with the indicated doses of CD39 ASO or with 100 $\mathrm{mg} / \mathrm{kg}$ of control oligo 1. Day 9 post ASO injection tumors were digested and CD39 expression on tumor infiltrating Tregs/non-Tregs (c), TAMs (d) and CD8 (e) was assessed by flow cytometry. Data is represented as fold-change in CD39 MFI compared to control oligo. In all cases each data point represents a mouse. Pooled data from two to three independent repeats. Error bars indicate SD. Asterisks indicate significant differences compared to control oligo 1 group

mCD39-specific ASO group were PD-1 positive (40\% vs. 96\%, Fig. 5 e, middle).

mCD39-specific ASO treatment combination with anti-PD1 antibody leads to anti-tumor efficacy

Targeting PD-1 on T cells represents a potential strategy to reinvigorate $\mathrm{T}$ cell function and offers a rational combination approach. We therefore tested the therapeutic efficacy of systemic CD39 ASO treatment in combination with anti-PD-1 antibody. To this end, we employed an orthotopic breast cancer tumor model by injecting EMT6 cells into the mammary gland (Fig. 6a). While treatment with $\mathrm{mCD} 39$-specific ASO resulted in a non-significant reduction in tumor size, combined treatment of mCD39-specific ASO and anti-PD-1 antibody led to significant reduction in tumor burden compared to vehicle control or mCD39-specific ASO monotherapy (Fig. 6b, c); one out of 12 mice rejected the tumor. These data provide a clinically relevant proof-of-principle for the therapeutic utility of CD39 targeting ASO which can be effectively combined with antibodies targeting PD-1.

\section{Discussion}

To further enhance the benefit of cancer immunotherapy, strategies targeting mechanisms of tumor immune evasion and/or resistance are of high clinical relevance. There is increasing evidence that extracellular adenosine generated by the ectonucleotidases CD39 and CD73 is a key metabolite that negatively regulates anti-tumor immunity [13]. In our study, we therapeutically target the expression of CD39 by ASOs. We demonstrate that CD39-specific ASOs reinstate ATP supply through potent and sustained suppression of CD39 expression on T cells, which improves T cell proliferation. Systemic treatment of CD39-specific ASO in vivo resulted in a substantial dose-dependent reduction (>50\%) of CD39 protein mainly in TAMs and Tregs, the latter correlating with an efficient reduction in Treg frequency. Improved tumor infiltrating $\mathrm{CD}^{+} \mathrm{T}$ cell to Treg density reflects reinvigorated anti-tumor immunity which could be 


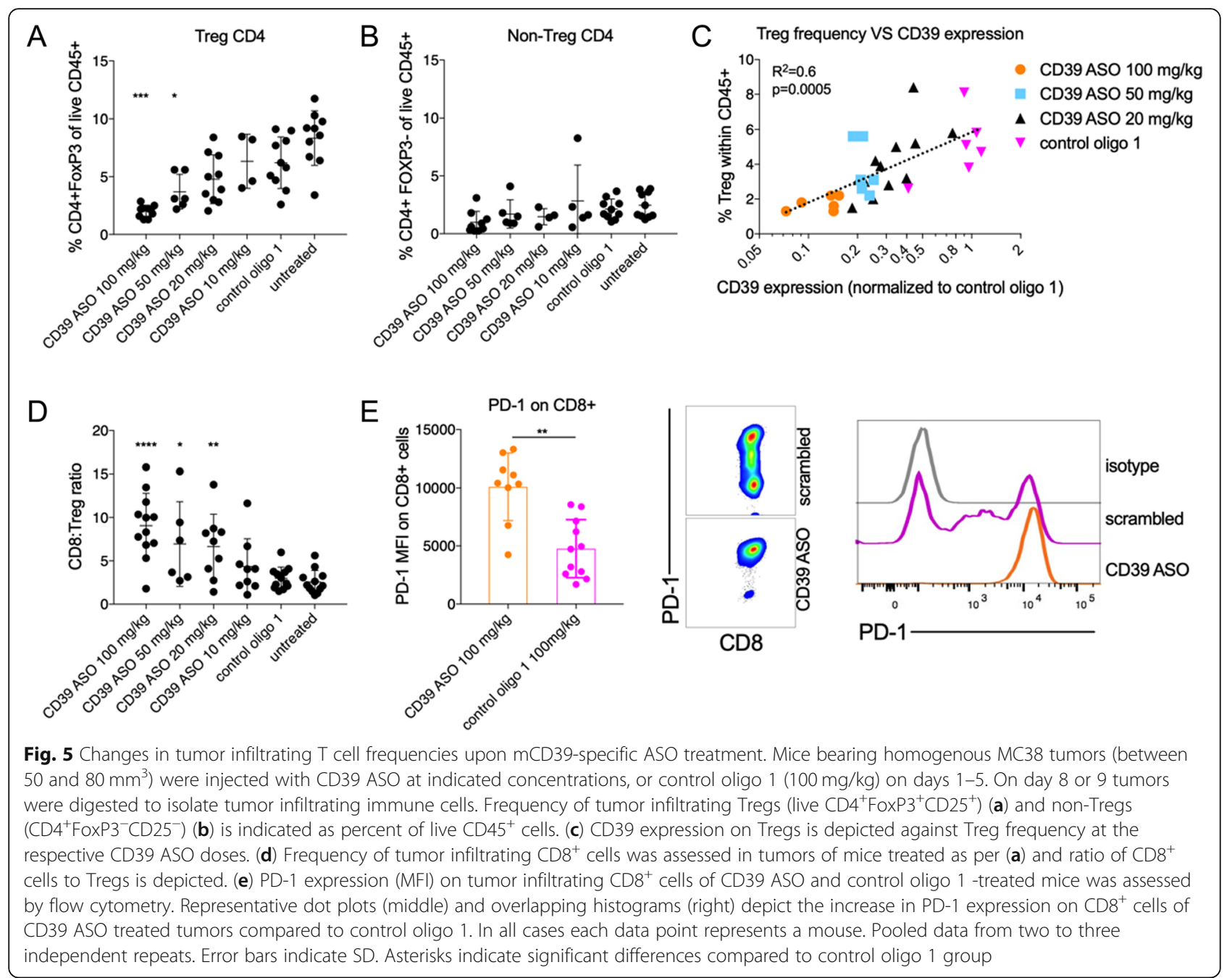

boosted by anti-PD-1 antibody to induce therapeutic efficacy.

Inhibitors of the CD39/CD73 pathway currently developed for clinical application are mainly composed of monoclonal antibodies and small molecule inhibitors [14]. We report here an entirely novel approach based on LNA Gapmer ASOs. While antibodies and small molecules may modulate the activity of already expressed targets, ASOs prevent the formation of the target protein by degradation of its mRNA. Chemically modified ASOs have enhanced stability and half-life, which may result in longer-lasting effects in vitro and in vivo [15]. Furthermore, ASOs exhibit high target specificity, which reduces side effects and due to their low molecular weight exhibit better tumor penetration.

We and others have demonstrated that tumor infiltrating $\mathrm{T}$ cells express high levels of CD39 in human and murine tumors [16, 17], and that the CD39-mediated release of excess adenosine generated from ATP leads to the suppression of $\mathrm{T}$ cell function in an autocrine manner. In agreement, we show that addition of extracellular ATP to CD39 expressing human $\mathrm{CD}^{+} \mathrm{T}$ cells considerably reduced their proliferation most likely due to the generation of adenosine by CD73, which can also be expressed by T cells [18]. In line with this, we demonstrate that human CD39-specific ASOs potently target CD39 expression on $\mathrm{T}$ cells in vitro and that the reduction of $\mathrm{T}$ cell proliferation was reverted by ASO-mediated suppression of CD39 expression. We therefore hypothesize that systemic treatment of cancer patients with CD39 ASO firstly could lead to reduced expression of CD39 in distinct tumor infiltrating immune cell populations. Secondly, this reduction in CD39 expression in turn can lead to a decreased degradation of ATP in the tumor microenvironment. As shown here in vitro, this could lead to increased proliferation of $\mathrm{CD}^{+}$effector $\mathrm{T}$ cells, thereby potentially improving anti-tumor $\mathrm{T}$ cell responses.

To validate our findings in vivo, we utilized an ASO specifically targeting mouse CD39 in immunocompetent murine tumor models. In agreement with published 


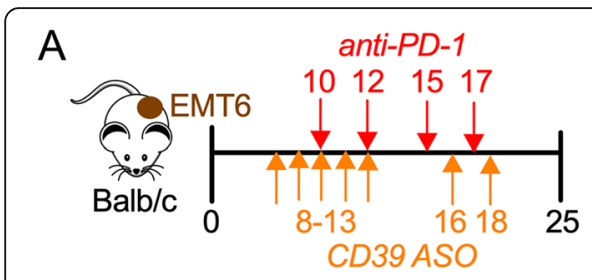

B
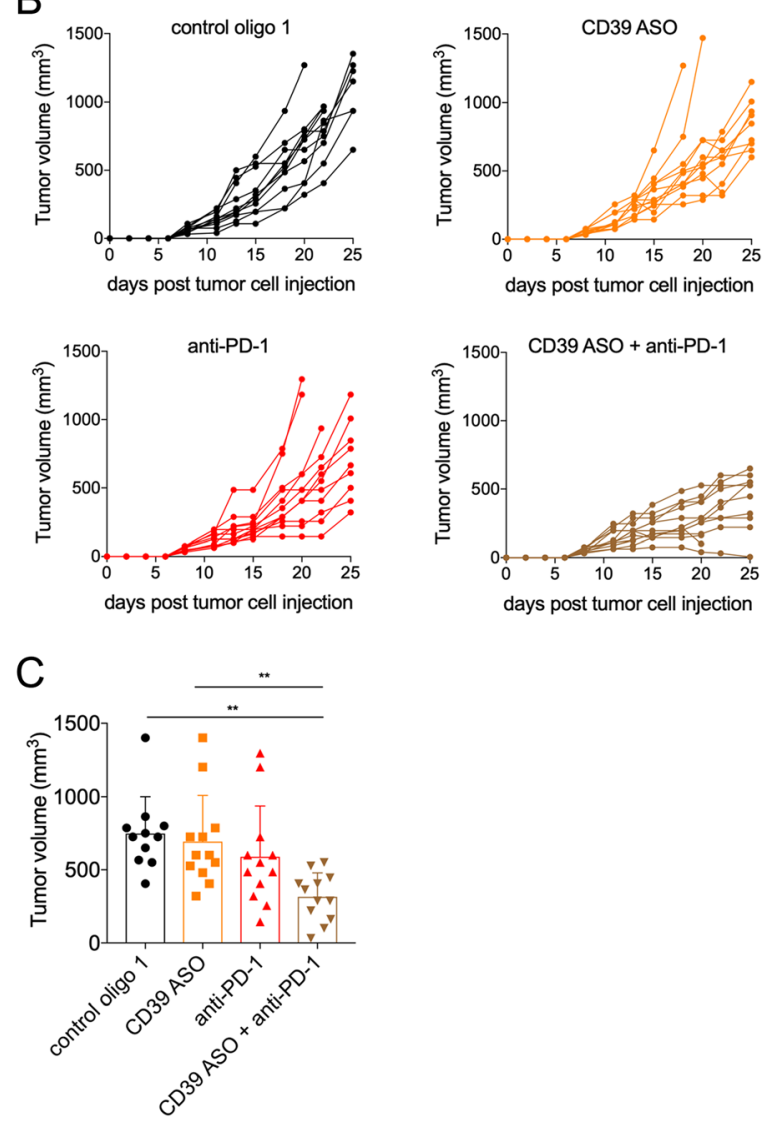

Fig. 6 Therapeutic efficacy of CD39 ASO in combination with antiPD-1 antibody. (a) Mice were injected intramammary with 250,000 EMT6 murine breast cancer cells. Once tumors reached $50-80 \mathrm{~mm}^{3}$ (Day 8) mice were injected with CD39 ASO, control oligo 1 and/or anti-PD-1 mAb (all i.p.) on indicated days. Mice were treated with CD39 ASO on five days in the first week and on two days in the second week. Tumor growth is depicted as spider plots (b) and as bar graph indicating cumulative tumor volume on day 25 post cell injection (c). Also included in (c) are euthanized mice reaching termination criteria (tumor volume above $1200 \mathrm{~mm}^{3}$ ) before day 25 . In all cases each data point represents a mouse. Pooled data from two independent repeats. Error bars indicate SD. Asterisks indicate significant differences compared to control oligo 1 group

findings [6, 19-21], CD39 was considerably expressed on a range of different tumor infiltrating immune cells. Of note, we noted the highest expression levels on TAMs, while tumor cells and B cells showed only moderate CD39 expression. Strikingly, systemic treatment of MC38 tumor bearing mice with a mouse-specific CD39 ASO potently suppressed CD39 protein expression on tumor infiltrating $\mathrm{CD}^{+}$Tregs and TAMs, but not on $\mathrm{CD}^{+} \mathrm{T}$ cells. Along the same line, the frequency of $\mathrm{T}$ cells in the tumor remained unchanged by CD39 ASO treatment. It is known that different cell types have different sensitivities to gymnotic delivery [11, 22], which may explain the observed differences in CD39 knockdown efficacy in different cell types. Indeed, additional extensive investigations are required to dissect cellular tropism and kinetics of ASOs in vivo. Furthermore, additional stimuli beyond CD39 inhibition may be necessary to provoke measurable infiltration with $\mathrm{T}$ cells as shown by others before [23, 24].

Tregs are well known to promote tumor progression by suppressing anti-tumor immunity [25, 26]. Various murine tumor models are known to be infiltrated by Tregs, and their depletion often improves anti-tumor immune responses [27-29]. Furthermore, increased numbers of Tregs correlate with poor prognosis in various types of human cancers $[26,30]$. Of note, Tregs in tumor-bearing hosts are known to express CD39 [31]. While almost all CD4 ${ }^{+}$CD25 $5^{+}$ cells in mice were reported to express high CD39 levels, only a subset of Foxp3 regulatory effector/memory-like T $\left(\mathrm{T}_{\mathrm{REM}}\right)$ cells stain positive for CD39 in humans [31]. Of particular interest, head and neck squamous cell carcinoma (HNSCC) patients were characterized by increased numbers of $\mathrm{CD}^{+} 9^{+}$Tregs, which hydrolyzed ATP at higher rates, and produced higher levels of adenosine than Tregs from healthy individuals [32]. This is consistent with data gained from tumor models in mice demonstrating that Tregs undergo apoptosis via oxidative stress in the tumor microenvironment and thereby release large amounts of ATP that is converted into adenosine via CD39 and CD73 [33]. In contrast, Tregs from CD39-knock-out (KO) mice failed to generate adenosine and were therefore unable to suppress proliferation of $\mathrm{CD}^{+}{ }^{+}$non-Treg cells in vitro [34]. In our study, ASO-mediated knockdown of CD39 correlated strongly with Treg frequencies in the tumor. While A2AR stimulation promotes the formation of FoxP3 ${ }^{+}$Treg [35, 36], CD39 inhibition may therefore, through reduced adenosine formation and decreased A2AR stimulation, reduce intratumoral Treg frequency after treatment with CD39-specific ASOs. Ultimately, this leads to a higher effector $\mathrm{CD}^{+} \mathrm{T}$ cell to Treg ratio, which is associated with improved clinical outcome [37]. We found that PD-1 and CD25 expression on $\mathrm{T}$ cells, which likely reflects $\mathrm{T}$ cell activation, was increased in mCD39-specific ASO treated animals. Although, assessment of additional markers is necessary to confirm $\mathrm{T}$ cell activation upon CD39 ASO treatment. Nevertheless, given the increased expression of PD-1 we speculated that a potential synergy with PD-1 blocking antibodies may be achieved through non-redundant but complementary mechanisms: CD39 ASOs antagonize CD39 expression, reduce Treg frequency and shift the balance towards effector $\mathrm{T}$ cells, the latter 
being further reinvigorated by PD-1 blockade. This strategy is currently pursued in early clinical trials in advanced solid tumors evaluating CD73 or A2a blockade in combination with PD-1/PD-L1 inhibitors (NCT02503774 and NCT02655822).

In conclusion, we demonstrate that ASOs targeting CD39 are able to achieve potent target suppression in relevant cell types in vitro and in vivo and induce potent anti-tumor effects in combination therapy with immune checkpoint inhibitors. Taken together, we developed an innovative immunotherapeutic tool that may potentially improve treatment options for cancer patients in the future.

\section{Additional files}

Additional file 1: Table S1. $I C_{50}$-values of selected hCD39 ASOs of first screening round in HDLM-2 cells (DOCX $13 \mathrm{~kb}$ )

Additional file 2: Figure S1. hCD39-specific ASO inhibit degradation of extracellular ATP in a human Burkitt's lymphoma cell line. (DOCX $662 \mathrm{~kb}$ )

Additional file 3: Table S2. Information on mouse-specific CD39 ASOs (DOCX $15 \mathrm{~kb}$ )

Additional file 4: Figure S2. $m C D 39$ specific ASOs show potent knockdown in the murine B cell lymphoma cell line A20. (DOCX 503 kb)

Additional file 5: Figure S3. CD39 expression on T cells and tumor cells after treatment with mCD39-specific ASO. (DOCX $918 \mathrm{~kb}$ )

Additional file 6: Figure S4. Intratumoral CD8+ T cell frequency and CD25 expression after treatment with mCD39-specific ASO. (DOCX 326 kb)

\section{Abbreviations \\ ASO: Antisense oligonucleotide; DC: Dendritic cells; G-MDSC: Granulocytic myeloid derived suppressor cell; LNA: Locked nucleic acids; M- MDSC: Monocytic myeloid derived suppressor cell; NK: Natural killer; TAM: Tumor associated macrophages; TDLN: Tumor draining lymph node; Treg: Regulatory T cells}

\section{Acknowledgements}

Not applicable

\section{Funding}

This work was supported by research grants from the Cancer League Switzerland KFS-3394-02-2014 (to AZ), Secarna pharamceuticals GmbH (to AZ) and the Federal Ministry of Education and Research; Förderkennzeichen: 031B0459 (to Secarna).

\section{Availability of data and materials}

All data generated or analysed during this study are included in this published article (and its additional files).

\section{Authors' contributions}

ASK, TT, RK, SMK designed and performed experiments and analyzed results; ASK, SMK and MB performed animal experiments; in vitro experiments were performed by $T$ T, RK, LH and MS; SM performed the bioinformatics analysis: FJ and AZ provided conceptual advice, generated and provided key reagents and wrote the paper; ASK, TT, RK, SMK, FJ, AZ designed the research and wrote the manuscript. All authors read and approved the final manuscript.

\section{Ethics approval}

All animal experiments were performed in accordance with Swiss federal regulations under animal ethics approval numbers 2408 and 2370. Collection of leukaphereses were performed under the ethics approval 329/16 S, Klinikum rechts der Isar, München.
Consent for publication

Not applicable

\section{Competing interests}

AZ received research funding from Secarna Munich, TT, RK, LH, MS, SM and FJ are employed by Secarna. The other authors have declared that no conflict of interests exists.

\section{Publisher's Note}

Springer Nature remains neutral with regard to jurisdictional claims in published maps and institutional affiliations.

\section{Author details \\ 'Cancer Immunology, Department of Biomedicine, University Hospital Basel, Basel, Switzerland. ${ }^{2}$ Secarna Pharmaceuticals GmbH, Planegg/Martinsried, Germany. ${ }^{3}$ Medical Oncology, University Hospital Basel, Basel, Switzerland. ${ }^{4}$ Present address: Novartis Institute of Biomedical Research, 4002 Basel, Switzerland.}

Received: 19 November 2018 Accepted: 22 February 2019 Published online: 12 March 2019

\section{References}

1. Bastid J, Cottalorda-Regairaz A, Alberici G, Bonnefoy N, Eliaou JF, Bensussan A. ENTPD1/CD39 is a promising therapeutic target in oncology. Oncogene. 2013:32(14):1743-51.

2. Di Virgilio F. Purines, purinergic receptors, and cancer. Cancer Res. 2012; 72(21):5441-7.

3. Antonioli L, Pacher P, Vizi ES, Hasko G. CD39 and CD73 in immunity and inflammation. Trends Mol Med. 2013;19(6):355-67.

4. Antonioli L, Blandizzi C, Pacher P, Hasko G. Immunity, inflammation and cancer: a leading role for adenosine. Nat Rev Cancer. 2013;13(12):842-57.

5. Beavis PA, Stagg J, Darcy PK, Smyth MJ. CD73: a potent suppressor of antitumor immune responses. Trends Immunol. 2012;33(5):231-7.

6. Bastid J, Regairaz A, Bonnefoy N, Dejou C, Giustiniani J, Laheurte C, et al. Inhibition of CD39 enzymatic function at the surface of tumor cells alleviates their immunosuppressive activity. Cancer Immunol Res. 2015;3(3):254-65.

7. Bonnefoy N, Bastid J, Alberici G, Bensussan A, Eliaou JF. CD39: a complementary target to immune checkpoints to counteract tumormediated immunosuppression. Oncoimmunology. 2015;4(5):e1003015

8. Salvestrini V, Orecchioni S, Talarico G, Reggiani F, Mazzetti C, Bertolini F, et al. Extracellular ATP induces apoptosis through P2X7R activation in acute myeloid leukemia cells but not in normal hematopoietic stem cells. Oncotarget. 2017:8(4):5895-908.

9. Sakaki H, Fujiwaki T, Tsukimoto M, Kawano A, Harada H, Kojima S. P2X4 receptor regulates $\mathrm{P} 2 \mathrm{X} 7$ receptor-dependent IL-1 beta and IL-18 release in mouse bone marrow-derived dendritic cells. Biochem Biophys Res Commun. 2013:432(3):406-11.

10. Frieden $\mathrm{M}$, Orum H. Locked nucleic acid holds promise in the treatment of cancer. Curr Pharm Des. 2008;14(11):1138-42.

11. Stein CA, Hansen JB, Lai J, Wu S, Voskresenskiy A, Hog A, et al. Efficient gene silencing by delivery of locked nucleic acid antisense oligonucleotides, unassisted by transfection reagents. Nucleic Acids Res. 2010;38(1):e3.

12. Jaschinski F, Korhonen $\mathrm{H}$, Janicot $\mathrm{M}$. Design and selection of antisense oligonucleotides targeting transforming growth factor Beta (TGF-beta) isoform mRNAs for the treatment of solid tumors. Methods Mol Biol. 2015; 1317:137-51.

13. Leone RD, Emens LA. Targeting adenosine for cancer immunotherapy. immunotherapy cancer. 2018;6(1):57.

14. Allard B, Longhi MS, Robson SC, Stagg J. The ectonucleotidases CD39 and CD73: novel checkpoint inhibitor targets. Immunol Rev. 2017;276(1):121-44.

15. Lindholm MW, Elmen J, Fisker N, Hansen HF, Persson R, Moller MR, et al. PCSK9 LNA antisense oligonucleotides induce sustained reduction of LDL cholesterol in nonhuman primates. Mol Ther. 2012;20(2):376-81.

16. Canale FP, Ramello MC, Nunez N, Araujo Furlan CL, Bossio SN, Gorosito Serran M, et al. CD39 expression defines cell exhaustion in tumor-infiltrating CD8(+) T cells. Cancer Res. 2018;78(1):115-28.

17. Thommen DS, Koelzer VH, Herzig P, Roller A, Trefny M, Dimeloe S, et al. A transcriptionally and functionally distinct PD-1(+) CD8(+) T cell pool with predictive potential in non-small-cell lung cancer treated with PD-1 blockade. Nat Med. 2018;24(7):994-1004. 
18. Bono MR, Fernandez D, Flores-Santibanez F, Rosemblatt M, Sauma D. CD73 and CD39 ectonucleotidases in T cell differentiation: beyond immunosuppression. FEBS Lett. 2015;589(22):3454-60.

19. Thelen M, Lechner A, Wennhold K, von Bergwelt-Baildon M, Schlosser HA. CD39 expression defines cell exhaustion in tumor-infiltrating CD8(+) T cellsletter. Cancer Res. 2018;78(17):5173-4.

20. d'Almeida SM, Kauffenstein G, Roy C, Basset L, Papargyris L, Henrion D, et al. The ecto-ATPDase CD39 is involved in the acquisition of the immunoregulatory phenotype by M-CSF-macrophages and ovarian cancer tumor-associated macrophages: regulatory role of IL-27. Oncoimmunology. 2016;5(7):e1178025.

21. Li J, Wang L, Chen X, Li L, Li Y, Ping Y, et al. CD39/CD73 upregulation on myeloid-derived suppressor cells via TGF-beta-mTOR-HIF-1 signaling in patients with non-small cell lung cancer. Oncoimmunology. 2017;6(6):e1320011.

22. Zhang Y, Qu Z, Kim S, Shi V, Liao B, Kraft P, et al. Down-modulation of cancer targets using locked nucleic acid (LNA)-based antisense oligonucleotides without transfection. Gene Ther. 2011;18(4):326-33.

23. Curran MA, Montalvo W, Yagita H, Allison JP. PD-1 and CTLA-4 combination blockade expands infiltrating $T$ cells and reduces regulatory $T$ and myeloid cells within B16 melanoma tumors. Proc Natl Acad Sci U S A. 2010;107(9): 4275-80.

24. Janakiram M, Pareek V, Cheng H, Narasimhulu DM, Zang X. Immune checkpoint blockade in human cancer therapy: lung cancer and hematologic malignancies. Immunotherapy. 2016:8(7):809-19.

25. Tanaka A, Sakaguchi S. Regulatory T cells in cancer immunotherapy. Cell Res. 2017:27(1):109-18.

26. Nishikawa H, Sakaguchi S. Regulatory T cells in cancer immunotherapy. Curr Opin Immunol. 2014;27:1-7.

27. Oh DS, Kim H, Oh JE, Jung HE, Lee YS, Park JH, et al. Intratumoral depletion of regulatory T cells using CD25-targeted photodynamic therapy in a mouse melanoma model induces antitumoral immune responses. Oncotarget. 2017;8(29):47440-53.

28. Maes W, Verschuere T, Van Hoylandt A, Boon L, Van Gool S. Depletion of regulatory $T$ cells in a mouse experimental glioma model through antiCD25 treatment results in the infiltration of non-immunosuppressive myeloid cells in the brain. Clin Dev Immunol. 2013;2013:952469.

29. Turk MJ, Guevara-Patino JA, Rizzuto GA, Engelhorn ME, Sakaguchi S, Houghton AN. Concomitant tumor immunity to a poorly immunogenic melanoma is prevented by regulatory T cells. J Exp Med. 2004;200(6):771-82.

30. Fridman WH, Pages F, Sautes-Fridman C, Galon J. The immune contexture in human tumours: impact on clinical outcome. Nat Rev Cancer. 2012;12(4): 298-306.

31. Borsellino G, Kleinewietfeld M, Di Mitri D, Sternjak A, Diamantini A, Giometto $\mathrm{R}$, et al. Expression of ectonucleotidase CD39 by Foxp3+ Treg cells: hydrolysis of extracellular ATP and immune suppression. Blood. 2007;110(4): 1225-32.

32. Mandapathil M, Szczepanski MJ, Szajnik M, Ren J, Lenzner DE, Jackson EK, et al. Increased ectonucleotidase expression and activity in regulatory T cells of patients with head and neck cancer. Clin Cancer Res. 2009;15(20):6348-57.

33. Maj T, Wang W, Crespo J, Zhang H, Wang W, Wei S, et al. Oxidative stress controls regulatory $\mathrm{T}$ cell apoptosis and suppressor activity and PD-L1blockade resistance in tumor. Nat Immunol. 2017;18(12):1332-41.

34. Deaglio S, Dwyer KM, Gao W, Friedman D, Usheva A, Erat A, et al. Adenosine generation catalyzed by CD39 and CD73 expressed on regulatory T cells mediates immune suppression. J Exp Med. 2007:204(6):1257-65.

35. Ohta A, Kini R, Ohta A, Subramanian M, Madasu M, Sitkovsky M. The development and immunosuppressive functions of CD4(+) CD25(+) FoxP3(+) regulatory T cells are under influence of the adenosine-A2A adenosine receptor pathway. Front Immunol. 2012;3:190.

36. Zarek PE, Huang CT, Lutz ER, Kowalski J, Horton MR, Linden J, et al. A2A receptor signaling promotes peripheral tolerance by inducing T-cell anergy and the generation of adaptive regulatory T cells. Blood. 2008;111(1):251-9.

37. Sato E, Olson SH, Ahn J, Bundy B, Nishikawa H, Qian F, et al. Intraepithelial CD8+ tumor-infiltrating lymphocytes and a high CD8+/regulatory $T$ cell ratio are associated with favorable prognosis in ovarian cancer. Proc Natl Acad Sci U S A. 2005:102(51):18538-43.

\section{Ready to submit your research? Choose BMC and benefit from:}

- fast, convenient online submission

- thorough peer review by experienced researchers in your field

- rapid publication on acceptance

- support for research data, including large and complex data types

- gold Open Access which fosters wider collaboration and increased citations

- maximum visibility for your research: over $100 \mathrm{M}$ website views per year

At BMC, research is always in progress.

Learn more biomedcentral.com/submissions 